\title{
Nerve Cable Graft Interposition in Patients with Brachial Plexus Schwannoma: Case Reports
}

\author{
Changsung Han, M.D., Hyo Yeong Ahn, M.D., Yeong Dae Kim, M.D., Chung Won Lee, M.D. \\ Department of Thoracic and Cardiovascular Surgery, Pusan National University Hospital, Pusan National University School of Medicine, Busan, Korea
}

\author{
ARTICLE INFO \\ Received May 4, 2021 \\ Revised June 21, 2021 \\ Accepted July 2, 2021 \\ Corresponding author \\ Hyo Yeong Ahn \\ Tel 82-51-240-7267 \\ Fax 82-51-243-9389 \\ E-mail doctorahn02@daum.net \\ ORCID \\ https://orcid.org/0000-0003-3198-8237
}

\begin{abstract}
Schwannomas are rare benign tumors that develop in Schwann cells lining peripheral nerves. Schwannomas of the brachial plexus are especially rare, accounting for $5 \%$ of all cases. Although several treatments can be considered, the exact method of treatment is unclear owing to the scarcity and sporadic occurrence of schwannomas. Tumor resection is performed in most cases, and nerve damage is inevitable in cases of neuroinvasive schwannoma. In this case series, we present our successful use of transposition of cable-grafted nerves for the treatment of schwannomas. We performed cable-grafted nerve interposition in addition to tumor resection, leading to increased recovery of nerve damage. To relieve postoperative symptoms and minimize sequelae, precise surgical tumor resection followed by nerve interposition using a cable-grafted nerve may be recommended.
\end{abstract}

Keywords: Brachial plexus, Schwannoma, Nerve transfer, Case report

\section{Case report}

\section{Case 1}

A 66-year-old woman was followed up for a schwannoma since 2013. As the schwannoma progressed, she presented with numbness and pain in the left infraclavicular region.

Muscle power and sensory integrity of the left upper limb were intact. Musculoskeletal contrast-enhanced magnetic resonance imaging (CE-MRI) of the thorax showed a swollen, well-defined, oval mass involving the brachial plexus, $5 \times 3.3 \mathrm{~cm}$ in size. The mass compressed and displaced the adjacent vessels.

An operation was performed under general anesthesia and an incision was made along the infraclavicular line. The minor pectoralis muscle was pulled laterally and the subclavian vessels were pushed down to approach the mass. We excised a well-encapsulated mass found at the location of the median nerve. Thereafter, we reconstructed the approximately $3.5-\mathrm{cm}$ nerve defect with a sural nerve cable graft from the left calf measuring approximately 9 $\mathrm{cm}$ in length. (Fig. 1A, B). For neurorrhaphy, simple inter- rupted sutures comprising approximately 8 stitches were performed using 8-0 and 9-0 Ethilon. The procedure was performed in the plastic surgery department and neuro-monitoring was not applied. A histopathological examination of the mass confirmed the diagnosis of schwannoma.

After the operation, the back of the hand and arm showed a decrease in sensory and muscle power. Starting on postoperative day (POD) 7, the muscle power progressively returned. Six months later, all sensory and muscle power had returned (Medical Research Council [MRC] score 5), and there was no evidence of recurrence.

\section{Case 2}

A 61-year-old man presented with a palpable mass in the left sternocleidomastoid (SCM) muscle and numbness in the left arm in October 2020.

A necrotic mass, approximately $24 \times 38 \mathrm{~mm}$ in size, was observed in the left supraclavicular area on neck computed tomography. We suspected that the mass was a metastatic lymph node, so we performed a core needle biopsy. However, a histopathological examination revealed a spindle 

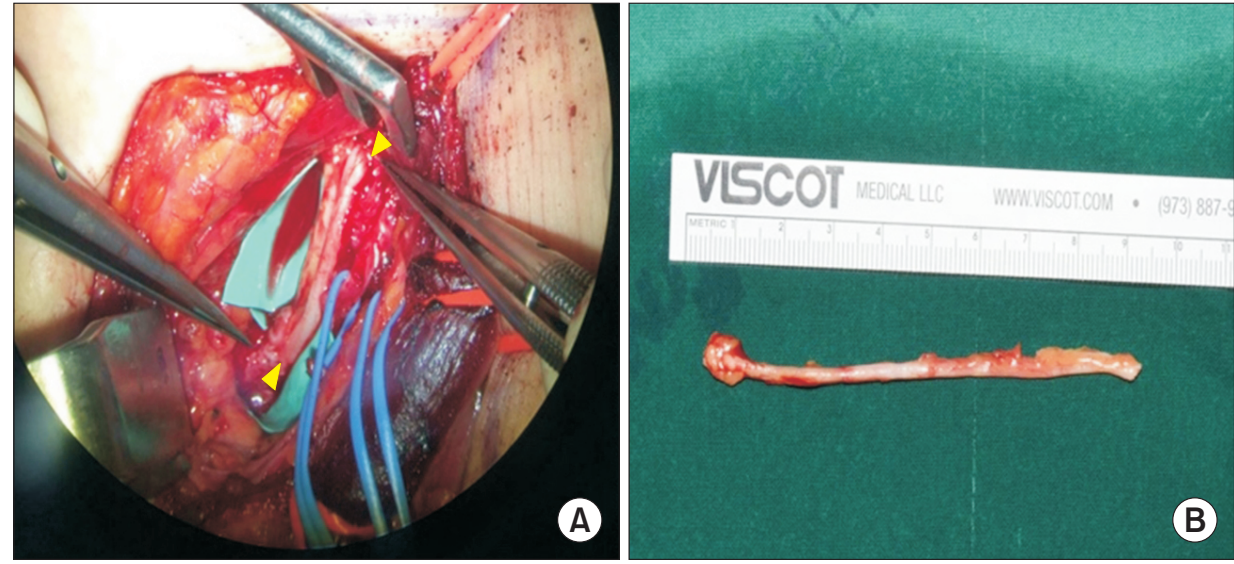

Fig. 1. (A) Sural nerve graft interposition in the brachial plexus (yellow arrowhead). (B) Sural nerve cable graft.
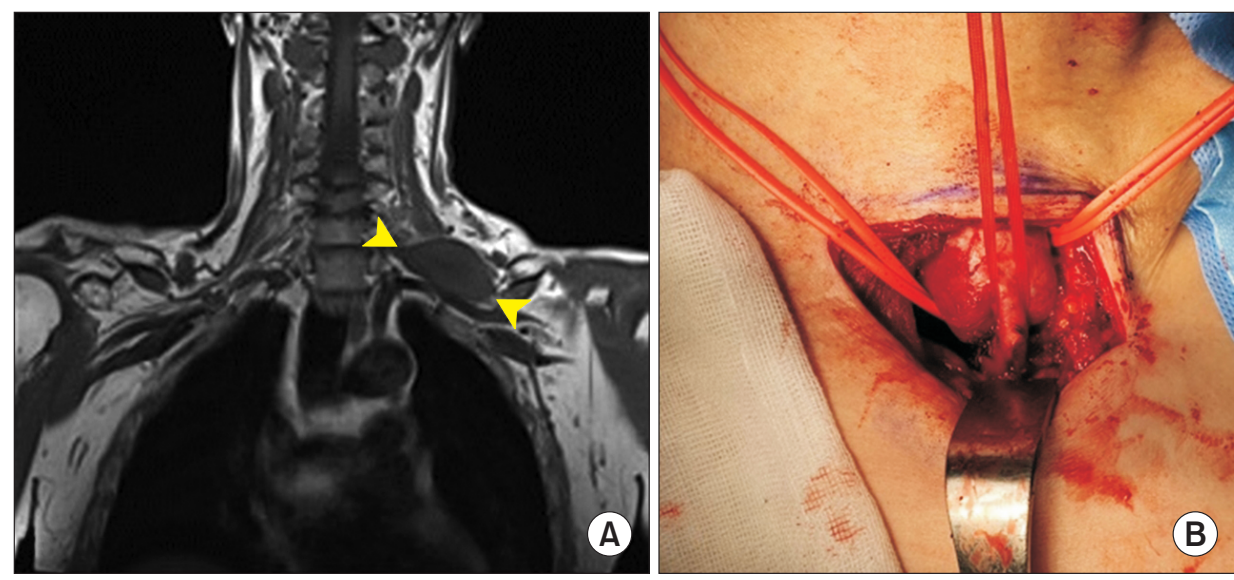

Fig. 2. (A) Magnetic resonance imaging showing schwannoma involving the brachial plexus (yellow arrowhead). (B) Well-encapsulated mass found in the brachial plexus.

cell lesion, suggestive of a neurogenic tumor (schwanno$\mathrm{ma})$, thus requiring resection.

The muscle power in the left arm was intact. Musculoskeletal CE-MRI of the thorax showed a swollen, well-defined, oval mass involving the brachial plexus, running along the $\mathrm{C} 8$ nerve and displacing the nerve root at $\mathrm{C} 7$ (Fig. 2A).

Under general anesthesia, the operation was performed through a supraclavicular incision, and the lateral side of the SCM muscle was dissected for approach. We excised a well-encapsulated mass located between the median and radial nerves (Fig. 2B). A nerve defect, approximately $6 \mathrm{~cm}$ in length, was interpositioned with a sural nerve cable graft from the left calf measuring approximately $8.5 \mathrm{~cm}$. The nerve graft end-to-end anastomosis was done by simple interrupted sutures (8-0 Ethilon). The process was cooperated by the plastic surgery department and nerve monitoring was not performed.

After the operation, the patient complained of decreased sensation and muscle power in the fourth and fifth fingers.
On POD 4, the patient was discharged and followed up closely. His upper arm motor function was evaluated in terms of the MRC score, and 3 months postoperatively, it returned to an MRC score of 4 .

\section{Case 3}

A 48-year-old man with a history of bladder cancer presented with a palpable left neck mass. The mass showed heterogeneous echogenicity and highly increased vascularity on ultrasonography. Therefore, we suspected that it was a metastatic nodule. First, we planned to perform an incisional biopsy under local anesthesia. However, when we started the incision, the patient complained of severe pain. On biopsy, the patient was diagnosed with schwannoma.

On musculoskeletal MRI, we observed a 3.6-cm enhanced mass arising from the level of division of the left brachial plexus (Fig. 3A). We suspected that it was a neurogenic tumor, requiring resection.

The operation was performed using a supraclavicular 

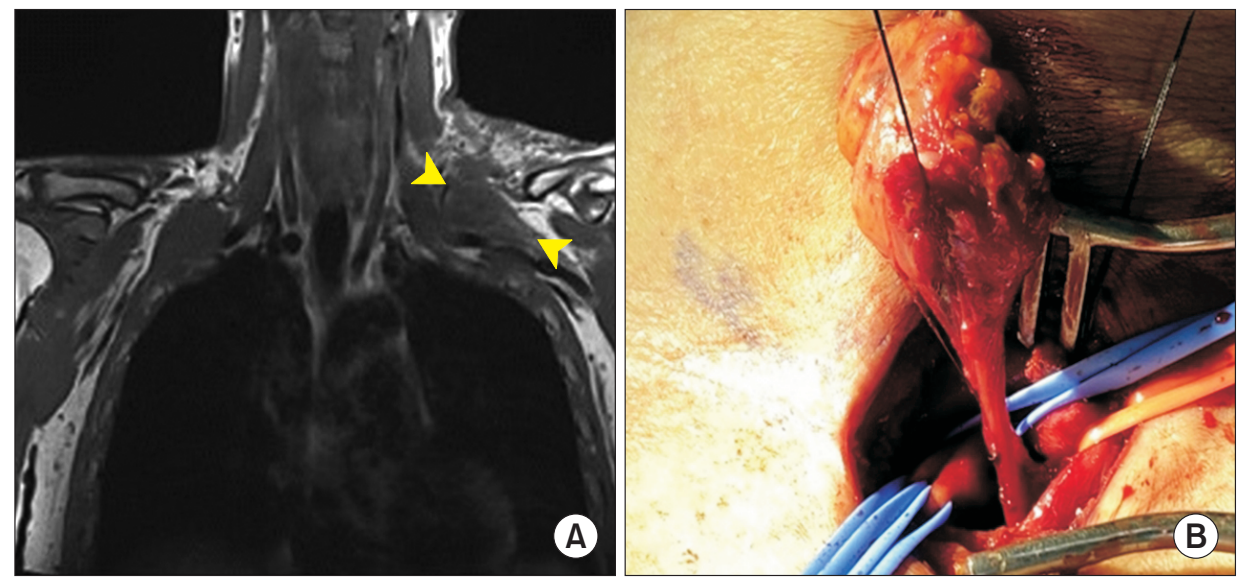

Fig. 3. (A) Magnetic resonance imaging (coronal view) showing postoperative tissue changes (yellow arrowhead). (B) A well-defined mass connected to the lower part of the nerve trunk.

approach and the incision was placed behind the SCM muscle under general anesthesia. A mass was found between the upper and lower trunks of the brachial plexus (Fig. 3B). In this case, it was difficult to detach the mass from the nerve trunk because the patient had previously undergone an incisional biopsy leading to postoperative tissue changes in the area. After tumor resection, nerve interposition was performed on the remaining nerve defect, measuring approximately $12 \mathrm{~mm}$, using a graft. The sural nerve cable graft measured approximately $27 \mathrm{~mm}$ and was harvested from the left calf. Neural cable implantation was done with simple interrupted sutures (8-0 Ethilon) was used. The nerve reconstruction was done by a plastic surgeon and neuro-monitoring was not performed.

During the in-hospital postoperative period, the patient complained of pain, rather than decreased sensation or muscle power of the arm. However, the pain improved over time, and the patient was discharged on POD 9. At the time of publication, the patient's upper arm motor function had returned to an MRC score of 2, but it is expected to recover to a score of 3 or 4 .

\section{Informed consent}

The patients in this study provided verbal informed consent for the publication of their clinical details and images.

\section{Discussion}

Schwannoma is a very rare disease that can occur anywhere in the peripheral nervous system. Schwannoma in the brachial plexus is especially rare, accounting for only $5 \%$ of all cases [1]. Generally, schwannomas are painless, and tumors smaller than $5 \mathrm{~cm}$ usually do not cause symp- toms. However, patients may present with pain or numbness secondary to adjacent nerve compression [2]. Most of these tumors are benign, and malignant transformation is extremely rare $[1,3]$.

Because most schwannomas are well encapsulated and can be saved without damaging the nerve, total surgical resection is the treatment of choice and results in a cure [3]. In rare cases where nerve invasion is observed, complete resection should be performed, and nerve damage is inevitable [4]. However, since the incidence of schwannoma itself is low and nerve damage is relatively uncommon, the optimal treatment method for schwannomas with nerve involvement is not clearly defined.

In the 3 patients described in this case series, nerve invasion by a schwannoma was observed, with a length of $>3$ $\mathrm{cm}$; thus, primary end-to-end nerve anastomosis after resection was impossible.

In cases of facial nerve injury, the sequelae after resection are significant. Therefore, many studies have been investigated ways to overcome nerve damage. If the resection defect is large, nerve interposition is performed by harvesting the sural nerve or the great auricular nerve with a cable nerve graft. Several studies have described nerve interposition of the facial nerve and reported recovery up to House-Brackmann (HB) grade 3 (the score for facial muscle motor evaluation), which was almost the same as the result of performing primary end-to-end anastomosis.

Therefore, we theorized that the same method could be applied to schwannoma nerve damage in the brachial plexus, and we reported 3 cases in which we successfully applied this method. To evaluate the results, the patient's upper limb motor and sensory parameters were checked postoperatively in terms of the MRC score. Similar to the $\mathrm{HB}$ grade of the facial nerve, nerve injury recovery was ob- 
served (i.e., the MRC score increased).

The representative postoperative complications after nerve graft interposition reported in several studies can be classified into 5 categories: sensory deficits, chronic pain, sensory symptoms (tingling, cold intolerance, paresthesia, or an irritating sensation), wound infection, and wound complications (hypertrophic scar, poor cosmesis, or wound dehiscence) $[5,6]$. In our cases, although a hypertrophic scar was observed in the first patient, no other significant complications that could affect daily life were reported in any of the 3 cases. Sensory deficits and pain at the surgical site recovered over time.

\section{Conflict of interest}

No potential conflict of interest relevant to this article was reported.

\section{Acknowledgments}

This work was supported by a 2-year research grant from Pusan National University.

\section{ORCID}

Changsung Han: https://orcid.org/0000-0003-3665-8982

Hyo Yeong Ahn: https://orcid.org/0000-0003-3198-8237

Yeong Dae Kim: https://orcid.org/0000-0001-7135-4648

Chung Won Lee: https://orcid.org/0000-0002-1160-6003

\section{References}

1. Vucemilo L, Lajtman Z, Mihalj J, et al. Brachial plexus schwannoma: case report and literature review. Acta Clin Croat 2018;57:36671.

2. Lee HJ, Kim JH, Rhee SH, Gong HS, Baek GH. Is surgery for brachial plexus schwannomas safe and effective? Clin Orthop Relat Res 2014:472:1893-8.

3. Rashid M, Salahuddin O, Yousaf S, Qazi UA, Yousaf K. Schwannoma of the brachial plexus; report of two cases involving the $C 7$ root. J Brachial Plex Peripher Nerve Inj 2013;8:12.

4. Huang JH, Samadani U, Zager EL. Brachial plexus region tumors: a review of their history, classification, surgical management, and outcomes. Neurosurg Q 2003;13:151-61.

5. Ducic I, Yoon J, Buncke G. Chronic postoperative complications and donor site morbidity after sural nerve autograft harvest or biopsy. Microsurgery 2020;40:710-6.

6. Ng SS, Kwan MK, Ahmad TS. Quantitative and qualitative evaluation of sural nerve graft donor site. Med J Malaysia 2006;61 Suppl B:13-7. 